\section{Standardisation et évaluation de la technique de salivation manuelle pour le dépistage des infections par trypanosomes chez la glossine (Diptera: Glossinidae)}

\author{
J.M. Kazadi ${ }^{1}$, M. Jochems ${ }^{1}$, H. Kabore ${ }^{1}$, C. \\ Mbeng ${ }^{1}$, J. Van Hees ${ }^{1}$, P. Kageruka ${ }^{1}$
}

KAZADI (J.M.), JOCHEMS (M.), KABORE (H.), MBENG (C.), VAN HEES (J.), KAGERUKA (P.). Standardisation et évaluation de la technique de salivation manuelle pour le dépistage des infections par trypanosomes chez la glossine (Diptera: Glossinidae). Revue Élev. Méd. vét. Pays trop., 1995, 48 (2) : 171-175

La méthode de salivation manuelle et celle de Bruce et al. sont décrites. Elles ont été évaluées simultanément, suivant un jeûne de 24,48 et 72 heures, sur 1702 mouches mâles non infectées, appartenant aux sousespèces de Glossina palpalis palpalis (Zaire), G. palpalis gambiensis (Bobo-Dioulasso), G. p. gambiensis (Maisons-Alfort) et $G$. morsitans morsitans (Mall). Le risque de salivation est de 0,66 pour la première méthode et de 0,01 pour la seconde. La standardisation de la méthode de salivation manuelle sur 79 mâles de G. m. morsitans (Mall) infectés avec Trypanosoma congolense IL 1180 a permis d'identifier 70,88 p. 100 des mouches porteuses d'infection mature et/ou immature. On note une nette différence entre les proportions des glossines qui salivent après un jeûne de $72 \mathrm{~h}$ et celles qui salivent après 48 et 24 heures.

Mots clés : Trypanosomose - Glossina - Salive.

\section{Introduction}

Dans les régions africaines qui constituent son aire de distribution, la mouche tsé-tsé, dont au moins 30 espèces et sous-espèces ont été répertoriées, vit dans des écosystèmes spécifiques où les conditions de milieu lui sont favorables. Ces endroits, désignés sous la dénomination anglaise de "fly-belts", montrent des étendues très limitées.

Dispersées et confinées dans les différentes zones zoogéographiques d'Afrique intertropicale, les glossines sont présentes dans 36 pays d'Afrique environ, infestant une surface évaluée à plus de 10 millions de $\mathrm{km}^{2}$ comprise entre $15^{\circ}$ de latitude nord et $21^{\circ}$ de latitude sud $(20,21)$, soit à peu près le tiers du continent, une région aussi vaste que les Etats-Unis d'Amérique.

Convoyée essentiellement par ces mouches, la trypanosomose sévit sur une surface comprise entre le tiers et la moitié de la superficie exploitable de l'Afrique ; elle a une incidence économique considérable du fait qu'elle oppose une barrière quasi infranchissable au développement agro-pastoral de nombreuses régions d'Afrique et maintient de grandes populations dans un état de malnutrition par carence en matières protéiques (2).

1. Institut de Médecine Tropicale Prince Léopold, département de Santé animale, Nationalestraat 155, B-2000 Antwerpen 1, Belgique.

Reçu le 13.3.1995, accepté le 14.9.1995
Des piqûres de glossines représentent un risque potentiel de la transmission cyclo-propagative de la trypanosomose humaine africaine (THA) et de la "nagana", respectivement chez l'homme et les animaux. Touré et Mortelmans (20) rappellent que la THA est, à ce jour, une endémie majeure à laquelle sont exposées environ 50 millions de personnes. La recrudescence de cette maladie dans les foyers de la vallée de Lambwe au Kenya (ICIPE cité par Youdeowei (24)), la montée de son indice de contamination nouvelle signalée au Zaïre (5), en Ouganda (16), au Congo (OMS cité par Laveissière et Hervouet (15)) et le taux croissant de son incidence chez les animaux (13) justifient l'importance qu'il faut accorder à la connaissance de la biologie de ce diptère.

Les trypanosomes de la section Salivaria montrent tous le mode de transmission cyclo-propagative chez la glossine : c'est par la salive que la mouche transmet les méta-trypanosomes infectieux. La salivation des glossines peut être provoquée pour recueillir la salive aux fins d'analyse physico-chimique et/ou dépistage des mouches porteuses d'infection mature ou immature de trypanosomes des sous-genres Trypanozoon, Nannomonas et Duttonella. A cet égard, différentes méthodes ont été utilisées $(4,6,24,25)$. Cependant, ces techniques fondées sur l'éthologie des mouches en quête de gorgement sont souvent laborieuses. Elles ont un faible rendement, exigent beaucoup de temps et parfois un équipement sophistiqué.

L'objectif de ce travail est de décrire une technique manuelle simple de salivation et de comparer son efficacité avec la méthode de Bruce et al. (4).

\section{Rappel anatomo-physiologique de l'appareil buccal}

Chez la glossine, les pièces buccales sont constituées des labium, labre et hypopharynx, dont l'ensemble forme l'haustellum ou proboscis. Au repos, cette fine et longue structure se trouve dirigée vers l'avant.

Situé à la base de la tête, le proboscis est engainé par les palpes maxillaires de même longueur que lui ; ces palpes se relèvent au moment de la piqûre, alors que le proboscis est abaissé verticalement (13).

Le milieu du labium est creusé par un sillon longitudinal qui loge l'hypopharynx. Cet organe est un long tubule qui est la continuation du conduit commun du canal salivaire; il repose dans la gouttière labiale et facilite l'écoulement de la salive.

L'épipharynx ou labre qui ferme la surface dorsale du labium forme une longue gouttière convexe, dont l'extrémité distale est effilée ; ses bords s'engrènent avec ceux de la gouttière labiale pour constituer le canal alimentaire à travers lequel le sang est aspiré pendant le repas.

Les glossines se caractérisent par le grand développement de leurs glandes salivaires dans la portion thoracoabdominale. L'importance qu'offre ici ces glandes témoigne du rôle joué par la sécrétion salivaire dans l'alimentation de ces diptères. C'est grâce à l'extrême abondance de cette sécrétion que le proboscis se trouve constamment humidifié et reste propre pendant les inter- 
valles de repas (22). Le rôle de la salive dans la physiologie de la digestion a été évoqué par Meyer (17). Roubaud (19) rappelle qu'il n'est pas inutile d'insister sur ces détails, parce qu'ils renferment en eux le secret du rôle spécifique des glossines dans l'évolution des trypanosomes pathogènes.

\section{Matériel et Méthodes}

\section{Méthode de salivation manuelle}

\section{Anesthésie des mouches}

Les glossines ( $n=25$ individus) maintenues dans des cages PVC (chlorure de polyvinyle) sont anesthésiées sous une cloche renfermant de l'azote. Après immobilisation, elles sont directement transférées dans des tubes individuels en polystyrène de $40 \mathrm{~mm}$ de hauteur sur 22 $\mathrm{mm}$ de diamètre interne et munis d'un bouchon à vis à l'une des extrémités. L'anesthésie induite par l'azote a une durée moyenne de trois minutes; au bout de cette période, les glossines reprennent leur activité en voltigeant maladroitement, tandis que leurs mouvements de vol et d'orientation se précisent.

\section{Capture et précautions}

On dévisse le bouchon avec précaution et introduit son index dans le flacon pour capturer la glossine entre le pli de la première articulation interphalangienne et la paroi du tube. Si cette manœuvre échoue, la mouche risque de saliver. Dans ce cas, les gouttelettes de salive sont perdues et il n'est pas évident de faire saliver la mouche une seconde fois.

L'introduction de l'index dans le tube ne permet que le passage de deux phalanges et leur taille s'oppose pratiquement à toutc escapade de la glossine. Lorsque la mouche est repêchée du tube, elle doit être immobilisée entre l'index et le pouce de l'opérateur. Seul l'haustellum demeure libre. Les pattes et les ailes doivent rester également contentionnées le long du corps de l'insecte pour éviter qu'elles n'essuient les gouttelettes de salive.

\section{Collecte du sang}

Une goutte de sang est prélevée à la queue d'une souris ou d'un rat sain. Elle est diluée dans un flacon contenant $3 \mathrm{ml}$ d'eau physiologique auxquels l'on ajoute une goutte d'héparine (Liquémine ${ }^{\circledR}$ ). Cette solution est maintenue sur de la carboglace durant toute la séance de salivation.

\section{Détection des trypanosomes}

Le sang dilué facilite la mise au point et la détection des trypanosomes contenus dans la salive. On dépose une goutte de cette dilution sur une lame porte-objet à laquelle on ajoute des gouttelettes de salive qui perlent au bout du proboscis. La préparation, couverte d'une lamelle $(18 \times 18 \mathrm{~cm})$, est observée au microscope à contraste de phase (x 400).

\section{Technique proprement dite}

En exerçant avec l'index et le pouce une pression dorsolatérale modérée sur la portion thoracique de la mouche, les palpes maxillaires se relèvent pour dégager la trompe. Les labre et labium libèrent l'extrémité proximale de l'hypopharynx qui élimine, à son tour, de fines gouttelettes de salive. Celles-ci sont déposées sur une lame porte-objet contenant une goutte de sang dilué comme décrit plus haut.

\section{Méthode de Bruce}

Bruce et al. (4) ont utilisé la méthode de salivation sur des lamelles maintenues sur une plaque chauffante portée à $37^{\circ} \mathrm{C}$. Burtt (6) a modifié cette méthode en substituant le cobaye à la place de la plaque chauffante et en faisant saliver les glossines sur des lames qui sont couvertes d'une couche d'ovo-albumine fixées à l'alcool absolu et colorées au Giemsa.

\section{Évaluation des méthodes de salivation manuelle et de Bruce}

\section{Chez les mouches non infectées}

La méthode de salivation manuelle et celle de Bruce et al. (4) ont été évaluées simultanément en soumettant les mouches à un jeûne de 24,48 ou 72 heures. Une population de mâles adultes non infectés ( $n=1702)$, composée des sous-espèces de Glossina palpalis palpalis (Zaïre), G. p. gambiensis (Bobo-Dioulasso), G. p. gambiensis (Maisons-Alfort) et $G$. morsitans morsitans (Mall), a été utilisée à cet effet. Cette dernière est une lignée sélectionnée génétiquement à partir de deux souches de glossines provenant de Kariba (Zimbabwe) et de Handeni (Tanzanie), dont la dénomination Mall est l'abréviation de morsitans allèles (8).

\section{Chez les mouches infectées}

Sur la base des bons résultats obtenus chez les mouches non infectées et soumises à un jeûne de 72 heures, la méthode de salivation manuelle a été standardisée sur des mâles $(n=79)$ de $G . m$. morsitans (Mall) infectés avec une souche de Trypanosoma congolense (IL 1180, clone dérivé de L 209) isolée sur un lion en Tanzanie.

\section{Analyse statistique des données}

Les résultats de ces deux méthodes ont été analysés statistiquement selon le modèle "loggods" suivant :

$\sum$ ayant salivé $+0,5$

$\log o d d s=\ln$ )

$\sum$ n'ayant pas salivé $+0,5$ 
TABLEAU I

Taux de salivation des glossines selon la durée de la diète (nouvelle méthode)

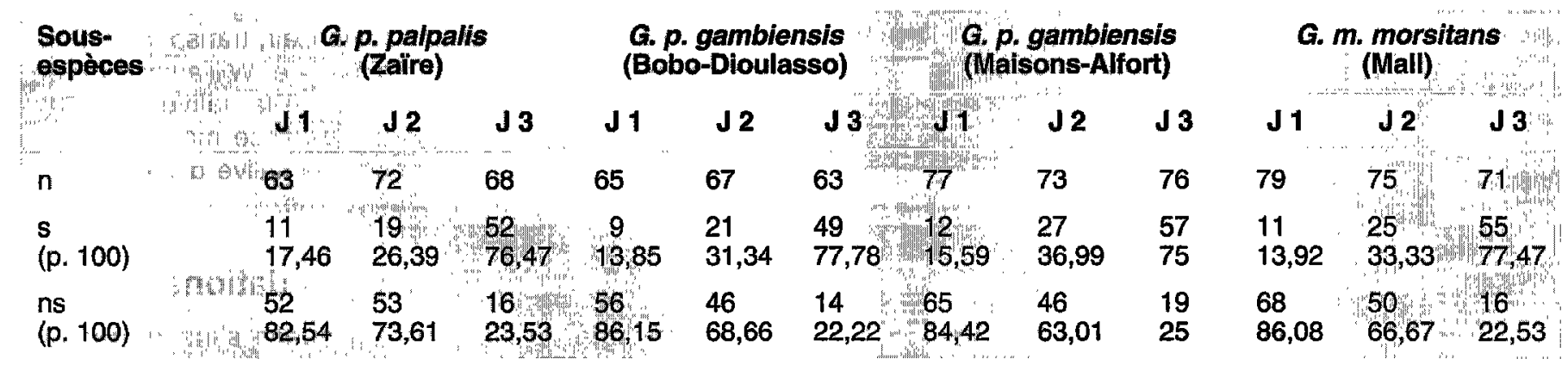

TABLEAU II

Taux de salivation des glossines selon la durée de la diète (méthode classique de Bruce)

\begin{tabular}{|c|c|c|c|c|c|c|c|c|c|c|c|c|}
\hline \multirow[t]{2}{*}{$\begin{array}{l}\text { Sous } \\
\text { especes }\end{array}$} & \multicolumn{3}{|c|}{$\begin{array}{l}\text { G. p. palpalls } \\
\text { (Zailre) }\end{array}$} & \multicolumn{3}{|c|}{$\begin{array}{c}\text { G. p. gambiensis } \\
\text { (Bobo-Dioulasso) }\end{array}$} & \multicolumn{3}{|c|}{$\begin{array}{l}\text { G. p. gambiensis } \\
\text { (Maisons-Alfort) }\end{array}$} & \multicolumn{3}{|c|}{$\begin{array}{l}\text { G. m. morsitans } \\
\text { (Mall) }\end{array}$} \\
\hline & J 1 & $\sqrt{2}$ & J 3 & J 1 & $\mathrm{~J} 2$ & J 3 & 01 & J 2 & J 3 & J1 & 12 & J 3 \\
\hline$n$ & 71 & 65 & 66 & 77 & 75 & 69 & 70 & 67 & 76 & 72 & 67 & 78 \\
\hline $\begin{array}{l}s \\
(p .100)\end{array}$ & $\begin{array}{l}0 \\
0\end{array}$ & $\begin{array}{l}1 \\
1,53\end{array}$ & $\begin{array}{l}0 \\
0\end{array}$ & $\begin{array}{l}3 \\
3,90\end{array}$ & $\begin{array}{l}0 \\
0\end{array}$ & $\begin{array}{l}2 \\
2,90\end{array}$ & 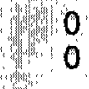 & $\begin{array}{l}0 \\
0\end{array}$ & $\begin{array}{l}1 \\
1,31\end{array}$ & $\begin{array}{l}0 \\
0\end{array}$ & $\begin{array}{l}0 \\
0\end{array}$ & $\begin{array}{l}2 \\
2,57\end{array}$ \\
\hline $\begin{array}{l}\text { ns } \\
(p .100)\end{array}$ & $\begin{array}{r}71 \\
100\end{array}$ & $\begin{array}{l}64 \\
98,47\end{array}$ & $\begin{array}{r}66 \\
100\end{array}$ & $\begin{array}{l}74 \\
96,10\end{array}$ & $\begin{array}{r}75 \\
100\end{array}$ & $\begin{array}{l}67 \\
97,10\end{array}$ & $\begin{array}{r}70 \\
100\end{array}$ & $\begin{array}{r}67 \\
100\end{array}$ & $\begin{array}{l}75 \\
98,69\end{array}$ & $\begin{array}{r}72 \\
100\end{array}$ & $\begin{array}{c}67 \\
100\end{array}$ & 96,93 \\
\hline
\end{tabular}

$\mathrm{n}=$ nombre $; \mathrm{s}=$ ayant salivé $; \mathrm{ns}=$ n'ayant pas salivé.

\section{Résultats}

La méthode manuelle montre qu'une mouche comprimée légèrement entre le pouce et l'index salive en moins d'une minute. Le tableau I rend compte de l'efficacité de cette méthode. II souligne, chez toutes les sous-espèces de glossines soumises à l'expérimentation, l'influence de la période de jeûne en relation avec le taux de salivation :

- le jeûne d'un jour montre un taux de 15,14 p.100, soit 43 mouches ayant salivé sur 284 individus :

- le jeûne de deux jours accuse un taux de 32,05 p.100, soit 92 mouches ayant salivé sur 287 individus ;

- le jeûne de trois jours révèle un taux de 76,61 p.100, soit 213 mouches ayant salivé sur 278 individus.

Considérés de manière globale, les résultats du tableau II mettent en évidence le faible rendement de la méthode de Bruce et al. (4). Nonobstant les modalités de la période de jeûne, le taux de salivation, qui est bas, s'élève à :

- 1,03 p.100 pour le jeûne d'un jour, soit 3 mouches ayant salivé sur 290 individus ;

- 0,36 p.100 pour le jeûne de deux jours, soit 1 mouche ayant salivé sur 274 individus ;

- 1,73 p.100 pour le jeûne de trois jours, soit 5 mouches ayant salivé sur 289 individus.

L'analyse statistique des données révèle qu'il existe une différence très significative entre les deux méthodes étudiées. Le risque de salivation des mouches est de 0,01 pour la méthode de Bruce et al. (4) et de 0,66 pour la méthode manuelle. La probabilité de salivation est consignée dans la figure 1.

La méthode de salivation manuelle montre une nette différence entre les proportions des mouches qui salivent après un jeûne de 72 heures et celles qui salivent après

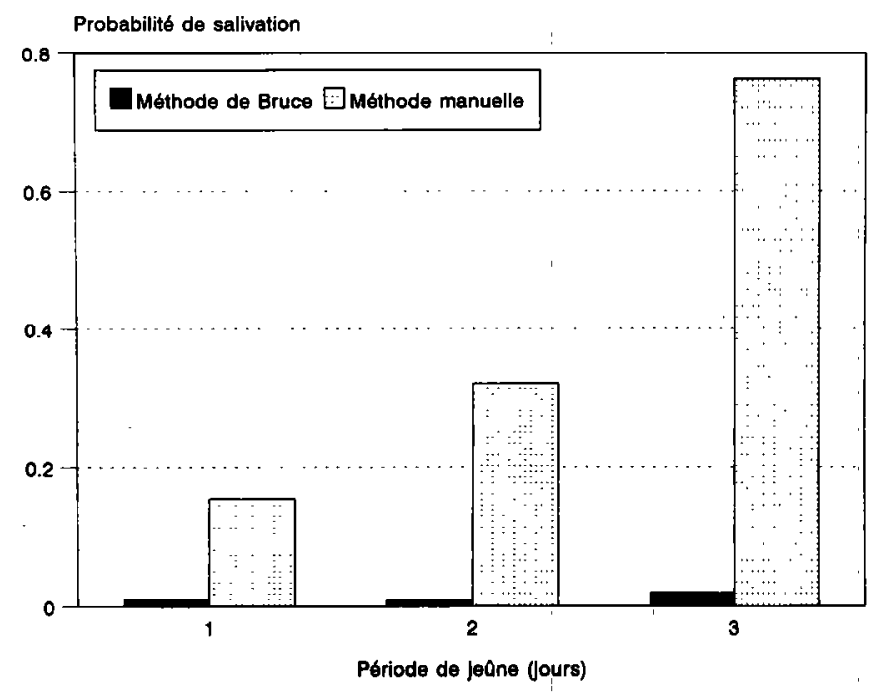

Figure 1: Comparaison de deux méthodes de sálivation. 
48 et 24 heures. L'observation microscopique révèle un taux de 70,88 p.100 des mouches infectées par $T$. congolense IL 1180 , soit 56 cas positifs sur 79 G. $m$. morsitans (Mall) examinées. Les trypanosomes serpentent aisément entre les érythrocytes : les métatrypanosomes sont observés en même temps que les formes mésocycliques, amastigotes, opisthosmastigotes et épimastigotes.

\section{Discussion}

La technique de salivation manuelle a l'avantage d'être directe et rapide. Elle met en relief l'influence de la période de jeûne sur la qualité des réponses des mouches à la salivation. Cette influence devient importante lorsque la période de jeûne augmente. Les résultats obtenus dans ce travail confirment les observations faites sur la salivation de G. austeni, G. morsitans et G. pallidipes. Plus les mouches ont faim, plus elles sont disposées à saliver ( 3 , 7,23 ). La méthode manuelle n'a pas de conséquence néfaste sur la survie des mouches. Aucune mortalité n'a été observée pendant et après l'expérimentation.

Le dépistage des formes mésocycliques évoluant à côté des formes métacycliques et épimastigotes permet de postuler que $T$. congolense suit une filière ascendante. Cette observation confirme ainsi la voie cylique décrite par Buxton (7). L'évaluation de la méthode manuelle semble indiquer que le processus de salivation est déclenché, lorsqu'une certaine pression est exercée légèrement sur la cage thoracique des glossines.

La physiologie sensorielle des tsé-tsé est mal connue ; si le rôle des antennes et des ommatidies est désormais élucidé, la fonction de nombreux autres organes de sens demeure encore obscure (1). Baldet et al. (1), Gnatzy et al. (12) ont reconnu que les sensilles campaniformes rassemblées sous la sous-costa et celles isolées le long de la nervure longitudinale I ont une fonction mécanoréceptrice. Les études de Ghysen (11) et celles de Palka et al.. (18) sur la drosophile ont montré que les mécanorécepteurs rassemblés sur la sous-costa réagiraient aux déformations de la cage thoracique et de la base de l'aile en excitant les neurones sensillaires qui se projettent sur la portion dorsale du ganglion nerveux thoracique.

La version originale ou modifiée de la méthode de Bruce et al. (4) a le grand inconvénient d'être passive, car audelà des stimuli environnementaux, le besoin de saliver semble dépendre de l'état physiologique de la mouche, notamment la faim.

Généralement, la minuscule goutte de salive excrétée est aussitôt dispersée par les pattes de la glossine ; elle sèche rapidement, puis cristallise et reste difficilement repérable. La mise en évidence des trypanosomes demande un effort supplémentaire de fixation et de coloration des traînées de cette salive. La perte de temps est d'autant plus significative que le nombre d'échantillons à examiner est grand.

L'observation macroscopique d'une goutte de salive montre que celle-ci est un liquide clair, transparent et floconneux. Williamson (23), Fairbairn et Williamson (9) ont étudié la composition chimique de la salive des glossines. On n'a relevé aucune différence macroscopique notable permettant de distinguer la salive d'une mouche infectée de celle d'une mouche saine.

\section{Conclusions et Recommandations}

La technique de salivation manuelle s'inscrit dans le cadre de la compétence vectorielle. Elle permet d'identifier les mouches porteuses d'infection mature ou immature et répond aux exigences de la mise au point d'une méthode fiable, simple et rapide de détection des trypanosomes chez une mouche vivante. Elle permettra d'évaluer ultérieurement le risque d'infection trypanosomienne chez les animaux traités préventivement ou curativement avec des trypanocides et soumis aux assauts glossinaires à des intervalles réguliers.

\section{Remerciements}

Les résultats présentés ici ont été soutenus financièrement par l'A.G.C.D. (Administration générale à la coopération et au développement, Gouvernement belge). Les auteurs adressent leur remerciement au Pr Dr S. Geerts pour avoir bien voulu accepter de lire et corriger ce manuscrit. IIs remercient également le Dr Ir. D. Berkvens pour l'analyse statistique des données ainsi que $\mathrm{Mme} \mathrm{Cl}$. Mattelaere pour le soin accordé à la mise en page de cet article.

\section{Bibliographie}

1. BALDET T., GEOFFROY B., D'AMICO F., CUISANCE D., BOSSY J.P., 1992. Structures sensorielles de l'aile de la glossine (Diptera: Glossinidae). Revue Élev. Méd. vét. Pays trop., 45 (3-4) : 295-302.

2. BLANC J.P., 1970. Les glossines : Méthodes de lutte. Thèse doct., Ecole Nationale Vétérinaire de Toulouse, France, $153 \mathrm{p}$

3. BRADY J., 1973. Changes in the probing responsiveness of starving tsetse flies (Glossina morsitans Westw.) (Diptera, Glossinidae). Bull. ent. Res., 63: 247-255.

4. BRUCE D., HARVEY D., HAMERTON A.E., DAVEY J.B., BRUCE R.R.C., 1914. Trypanosome diseases of domestic animals in Nyassaland. I. Trypanosoma simiae sp. Nov. Part III. Rep. Sleep. Sickn. Commn. R. Soc., 15: $137,138,141$.

5. Bureau central de la trypanosomiase, 1991. Rapport annuel d'activités 1990. Kinshasa, Zaïre, Bureau central de la trypanosomiase.

6. BURTT E., 1946. Salivation by Glossina morsitans on to glass slides: a technique for isolating infected flies. Ann. trop. Med. Parasit., 40: 141-144.

7. BUXTON P.A., 1955. The natural history of tsetse flies. London, U.K., H.K. Lewis and Co. Ltd, 816 p. (Memoir No.10 of London school of hygiene and tropical medicine) 
8. ELSEN P., VAN HEES J., DE LIL E., 1993. L'historique et les conditions d'élevage des lignées de glossines (Diptera, Glossinidae) maintenues à l'Institut de Médecine tropicale Prince Léopold d'Anvers. J. Afr. Zool, 107: 439-449.

9. FAIRBAIRN H., WILLIAMSON J., 1956. The composition of tsetse-fly saliva. I. A histo-chemical analysis. Ann. trop. Med. Parasit., 50: 322-333.

10. FREZIL J.L., 1983. La trypanosomiase humaine en République Populaire du Congo. Paris, France, ORSTOM, 165 p. (Trav. Doc. ORSTOM $n^{\circ} 155$ )

11. GHYSEN A., 1978. Sensory neurones recognise defined pathways in Drosophila central nervous system. Nature, 274: 869-872.

12. GNATZY W GRINERT U., BENDER M., 1987. Campaniform sensilla of Calliphora vicina (Insecta, Diptera). I. Topograpy. Zoomorph., 106: 312-319.

13. ITARD J., 1986. Les glossines ou mouches tsé-tsé. Maisons-Alfort France, IEMVT, 155 p. (Etudes et synthèses de l'IEMVT n ${ }^{\circ} 15$ )

14. JORDAN A.M., 1986. Trypanosomiasis control and African rural Development. London, U.K., Longman, 367 p.

15. LAVEISSIERE C., HERVOUET J.P., 1991. La trypanosomiase humaine en Afrique de l'Ouest. Paris, France, ORSTOM Editions, 147 p.

16. MBULAMBERI D.B., 1990. Recent epidemic outbreaks of human trypanosomiasis in Ouganda. Insect Sci. Applic., 11: 289-292.

17. MEYER P., 1977. Physiologie humaine. Paris, France, Flammarion Médecine-Sciences, $1320 \mathrm{p}$.

18. PALKA J., MALONE M.A., ELLISON R.L., WIGSTON D.J., 1986. Central projections of identified Drosophila sensory neurons in relation to their time of development. J. Neurosci., 6: 1822-1830.

19. ROUBAUD E., 1909. La Glossina palpalis. Sa biologie, son rôle dans l'étiologie des trypanosomiases. In : Martin, Leboeuf, Roubaud eds, Rapport de la mission d'ćtudes de la maladie du sommeil au Congo français, 1906 - 1908. Paris, France, Masson, $280 \mathrm{p}$
20. TOURÉ S.M., MORTELMANS J., 1990. Impact de la trypanosomose animale africaine (TAA). Bull. Séanc. Acad. r. Sci. outre Mer, 36 : 239257.

21. TRONCY P.M., ITARD J., MOREL P.C., 1981. Précis de parasitologie vétérinaire tropicale. Paris, France, ministère de la Coopération et du Développement, 717 p. (Manuels et Précis d'élevage ${ }^{\circ} 10$ )

22. WIGGLESWORTH V.B., 1972. The principles of insect physiology. London, U.K., Chapman and Hall, $827 \mathrm{p}$

23. WILLIAMSON J., 1956. The composition of tsetse-fly saliva. II. Analysis of amino acids and sugar by paper partition chromatography. Ann. trop. Med. Parasit., 50: 334-344.

24. YOUDEOWEI A. 1975. A simple technique for observing and collecting the saliva of tsetse flies (Diptera, Glossinidae). Bull. ent. Res., 65: 6567.

25. YOUDEOWEI A., 1976. Salivary secretion in Wild Glossina pallidipes Austen (Diptera, Glossinidae), Acta trop., 33 (4): 369-375.

KAZADI (J.M.), JOCHEMS (M.), KABORE (H.), MBENG (C.), VAN HEES (J.), KAGERUKA (P.). Standardisation and evaluation of a manual salivation technique for the detection of trypanosome infections in tsetse fly (Diptera: Glossinidae). Revue Élev. Méd. vét. Pays trop., 1995, $48(2)$ : 171-175

Two methods of salivation of tsetse flies, namely manual salivation and method of Bruce et al. were simultaneously evaluated on 1,702 male uninfected Glossina palpalis palpalis (Zä̈re), G. palpalis gambiensis (Bobo-Dioulasso), G. p. gambiensis (Maisons-Alfort) et G. morsitans morsitans (Mall) fasted for 24, 48 and 72 hours. The risk of salivation was 0.66 by the manual method and 0.01 by the method of Bruce et al. The manual salivation method was standardised on 79 male G. m. morsitans (Mail) infected with Trypanosoma congolense IL 1180. By this method, $70.88 \%$ of flies carrying mature and/or immature infection were identified. A clear difference was observed in the proportion of tsetse flies which salivated after 72 hours and those which salivated after 48 and 24 hours of fasting.

Key words: Trypanosomosis - Glossina - Saliva. 